\title{
Promotive effects of capillary morphogenetic protein 2 on glioma cell invasion and the molecular mechanism
}

\author{
Yifan Xu, Yuxin He, Wu Xu, Tianyu Lu, Weibang Liang, Wei Jin \\ Nanjing Drum Tower Hospital, The Affiliated Hospital of Nanjing University Medical School, Nanjing, Jiangsu Province, P.R. China
}

\begin{abstract}
We aimed to explore the role of capillary morphogenetic protein 2 (CMG2) in glioma cell invasion and the possible molecular mechanism. Glioma cells U87 and U251 stably overexpressing CMG2 were constructed by lentiviral transfection. The changes of cell invasion and migration were tested by Matrigel-transwell assay and scratch assay, respectively. A mouse model with orthotopically transplanted tumour was established to evaluate the effects of CMG2 overexpression on the in vivo invasion of glioma cells and survival time. The differences of filopodia and lamellar pseudopodia among glioma cells with different CMG2 expressions were observed by immunofluorescence assay. The expressions of YAP and $p$-YAP in glioma cells overexpressing CMG2 or not were compared by Western blot. Compared with the control group, overexpression of CMG2 enhanced the invasion and migration capacities of glioma cells $(p<0.05)$. The tumour tissues of mice transplanted with glioma cells overexpressing $C M G 2$ were obviously invaded, and their survival time was significantly shortened ( $p<0.05$ ). Immunofluorescence staining showed that glioma cells overexpressing CMG2 formed more lamellipodia and filopodia than those of the control group. As glioma cells overexpressing CMG2 formed more pseudopodia, the expression of YAP, a key effector protein of the Hippo pathway, was up-regulated. CMG2 promoted the invasion of glioma cells, and may induce pseudopodium formation by up-regulating YAP expression.
\end{abstract}

Key words: glioma, invasion, capillary morphogenetic protein 2, YAP.

\section{Introduction}

Originating from glial cells, glioma is the most common primary tumour of the central nervous system. According to the classification criteria of WHO, it can be classified into I-IV grades, of which grades I-II correspond to low grade and grades III-IV correspond to high grade, with grade IV glioma being most malignant [17]. Due to strong invasive ability, malignant glioma cannot be completely removed by surgery. Meanwhile, the lack of effective drugs after surgery may easily cause recurrence, which results in poor prognosis and seriously affects the quality of life [5]. Therefore, it is of great significance to further understand the mechanisms involved in the onset and progression of glioma, especially related factors responsible for the significant invasion of glioma cells, aiming to find effective therapeutic targets.

Capillary morphogenetic protein 2 (CMG2) was discovered because of its high expression during angiogenesis, and thereafter identified as an anthrax toxin receptor that can mediate the entrance of anthrax toxins into host cells to exert cytotoxic effects. Thus, CMG2 is also referred to as anthrax toxin receptor 2 [4]. Until now, the physiological functions of CMG2 have still been elusive. CMG2

\section{Communicating authors}

Weibang Liang, Wei Jin, Nanjing Drum Tower Hospital, The Affiliated Hospital of Nanjing University Medical School, Nanjing 210000 , Jiangsu Province, P.R. China, e-mail: liangweibangdn@sina.com, jinwei_dn@sina.com 
mutations can cause juvenile hyaline fibromatosis. Dowling et al. found that CMG2 was highly expressed in glioma, and positively correlated with the glioma grade but negatively correlated with prognosis [6], suggesting that CMG2 may play an important role in the onset and progression of this tumour. It is well established that the crucial morphological basis of tumour cell invasion is the formation of pseudopodia. El Kamah et al. reported that CMG2 facilitated the formation of laminar pseudopodia and filopodia in glioma cells [7]. Actually, the formation of pseudopodia of tumour cells is a complex molecular regulation process which involves several signalling pathways, including the Hippo pathway.

Abnormal expression of YAP, a key effector molecule in the Hippo signalling pathway, has been found in various tumours such as liver cancer, lung cancer and breast cancer [10]. Also, the Hippo/YAP signalling pathway often becomes disordered in glioma [29]. YAP can promote the invasion and metastasis of gastric cancer, non-small cell lung cancer, pancreatic cancer and breast cancer $[12,13,23,25]$. For primary renal carcinoma cells, knocking down the expression of YAP can reduce the formation of pseudopodia and their migratory ability, suggesting that YAP may affect the invasion and migration of tumour cells by influencing pseudopodium formation [20]. However, whether YAP participates in the regulation of pseudopodium formation in glioma cells and promotes their invasion has never been studied hitherto. The aim of this study was to explore the role of CMG2 in glioma invasion and the possible molecular mechanism. The findings provide a new index for determining the prognosis of glioma and a novel target for clinical treatment.

\section{Material and methods \\ Cells, mice and reagents}

Glioma cell lines U87 and U251 were purchased from Shanghai Beinuo Bioscience Co., Ltd. (China). Four- to six-week-old male immunodeficient mice were bought from Beijing Vitalstar Biotechnology Co., Ltd. (China). Antibodies against CMG2, GAPDH, YAP and p-YAP were obtained from Cell Signaling Technology (USA). Foetal bovine serum (FBS) was provided by Hangzhou Sijiqing Biological Engineering Materials Co., Ltd. (China). Trypsin was purchased from Beyotime Institute of Biotechnology Co., Ltd. (China). WesternBright ECL HRP colour development solution, FITC-phalloidin and fluorescent secondary antibody were bought from Sigma (USA). SDSPAGE rapid preparation kit was obtained from BioRad (USA). DAB kit was supplied by Fuzhou Maixin Biotechnology Development Co., Ltd. (China). Triton X-100 was purchased from Beijing Solarbio Science \& Technology Co., Ltd. (China).

\section{Ethics}

All animal experiments have been approved by the ethic committee of our hospital, and performed in conformance with the legal requirements in Poland as well as with the European Communities Council Directive of 24 November 1986 or the National Institute of Health Guide (National Institute of Health Publications No. 80-23, Revised 1978) for the care and use of Laboratory Animals for experimental procedures.

\section{Cell culture}

Glioma cell lines U87 and U251 were taken from liquid nitrogen and dissolved at $37^{\circ} \mathrm{C}$ in a water bath. The cells were added with DMEM containing 10\% FBS. The supernatant was removed by centrifugation, and then the cells were added with culture medium for suspension, inoculated in a culture flask and cultured at $37^{\circ} \mathrm{C}$ with $5 \% \mathrm{CO}_{2}$. When the confluence reached $80 \%$, the cells were digested with $0.25 \%$ trypsin, and passaged with complete medium.

\section{Construction of glioma cell line stably overexpressing CMG2}

The coding region of CMG2 (NM_001145794) was loaded into a lentiviral expression vector and packaged by Shanghai Genechem Co., Ltd. (China). Well-growing cells were counted and inoculated in a Petri dish until the confluence was about $40 \%$. Then the cells were transfected with control lentivirus and overexpression lentivirus, and the culture medium was replaced with DMEM complete medium containing $10 \%$ FBS after $24 \mathrm{~h}$. Forty-eight hours after transfection, the transfection efficiency was observed under a fluorescence microscope. Successfully transfected glioma cells showed green fluorescence under the microscope due to GFP expression, i.e. the vector protein. Afterwards, positive cells were screened by using puromycin and flow cytometer. Interference efficiency was measured by RT-PCR and Western blot. 


\section{Scratch assay}

An appropriate amount of glioma cells were inoculated into a 24-well plate. When the confluence reached approximately $90 \%$, straight scratches with a uniform thickness were made using a pipette tip. After repeated rinsing with PBS until no cells suspended, PBS was discarded and $500 \mu \mathrm{l}$ of low-serum medium (DMEM medium containing 1\% FBS) was added to each well. Finally, cell migration was observed.

\section{Transwell assay}

Transwell chambers were added into a 24-well plate. An appropriate amount of Matrigel gel was mixed with pre-cooled DMEM at a ratio of $1: 1$, and spread onto the upper chamber (10 $\mu \mathrm{l} /$ chamber). Then the chamber was placed at $37^{\circ} \mathrm{C}$ for $30 \mathrm{~min}$, and cells were digested, resuspended, and diluted with culture medium to a concentration of $1 \times 10^{5} / \mathrm{ml}$. Subsequently, $500 \mu$ l of $10 \%$ FBS was added into the lower chamber. After $200 \mu$ l of cell suspension was pipetted from each group into the upper chamber, the 24-well plate was cultured in a $37^{\circ} \mathrm{C}$ incubator. After $24 \mathrm{~h}$, fluid in the chamber was discarded and the cells were washed 3 times with pre-warmed PBS. After being fixed with $4 \%$ paraformaldehyde for $20 \mathrm{~min}$, the cells on the chamber membrane were washed 3 times with PBS ( 5 min each time), and thereafter stained with crystal violet for 5 min. The chamber was rinsed with tap water, and then the cells on the lower surface of the chamber membrane were carefully wiped off with a cotton swab and observed under a microscope after drying.

\section{RT-PCR}

After cells were transfected for $48 \mathrm{~h}$, the culture medium in a 6-well plate was discarded, and $1 \mathrm{ml}$ of pre-cooled Trizol reagent was added to each well. The cells were thoroughly mixed, placed on ice for $5 \mathrm{~min}$, added with $0.2 \mathrm{ml}$ of chloroform, shaken, left still at room temperature for $5 \mathrm{~min}$, centrifuged to collect the aqueous phase into an EP tube, added with isopropanol, shaken on ice for $5 \mathrm{~min}$, centrifuged again to discard the supernatant, mixed with $75 \%$ ethanol solution, and finally centrifuged to discard the supernatant. After the precipitate was dried, the cells were mixed with 20-50 $\mu$ l of DEPC-water and tested for purity. Afterwards, total RNA was reversely transcribed into cDNA according to the instruc- tions of the PrimeScrip reverse transcription kit. The PCR reaction system was prepared according to the SYBR Premix Ex Taq instructions. The reaction conditions were $95^{\circ} \mathrm{C}$ for $30 \mathrm{~s}, 1 \mathrm{cycle} ; 95^{\circ} \mathrm{C}$ for $5 \mathrm{~s}$ and $60^{\circ} \mathrm{C}$ for $30 \mathrm{~s}, 40$ cycles; $95^{\circ} \mathrm{C}$ for $5 \mathrm{~s}$ and $60^{\circ} \mathrm{C}$ for $1 \mathrm{~min}, 1$ cycle. Using $\beta$-actin as an internal reference, the relative expression level was represented as 2- $\triangle \Delta C T$. CMG2 sequences: F: 5'-CAAAATCAGTAAAGGCTTGG-3'; R: 5'-CAAAGGTTCTTCTTCCTCCT-3'. $\beta$-Actin sequences: $F:$ 5'-CCTCGCCTTTGCCGA-3'; R: 5'-GCCCACATAGGAATCCTTC-3'.

\section{Western blot}

After being transfected for $48 \mathrm{~h}$, cells were added with an appropriate amount of RIPA lysate, lysed for $30 \mathrm{~min}$, and centrifuged at $12,000 \mathrm{rpm}$ and $4^{\circ} \mathrm{C}$ for $10 \mathrm{~min}$, from which the supernatant was collected. The protein concentration was measured using BCA kit and the protein sample was mixed with loading buffer. After denaturation in $100^{\circ} \mathrm{C}$ water for $5 \mathrm{~min}$, SDS-PAGE was conducted, and the products were electronically transferred onto a PVDF membrane. Subsequently, the membrane was blocked for $2 \mathrm{~h}$ using 5\% skimmed milk, incubated with primary antibodies against CMG2 (1: 1000 diluted), YAP (1: 500 diluted), p-YAP (1: 500 diluted) and GAPDH (1: 5000 diluted) overnight at $4^{\circ} \mathrm{C}$, washed by TBST, incubated with HRP-labelled goat anti-rabbit IgG at $37^{\circ} \mathrm{C}$ for $2 \mathrm{~h}$, and added with ECL for colour development. Images were acquired using an automated gel imaging system, and protein expression levels were analysed with GAPDH as an internal reference.

\section{Cytoskeleton fluorescent staining}

Sterile cover glasses were placed in a 24-well plate, coated with an appropriate amount of polylysine for $30 \mathrm{~min}$, and washed with sterile PBS three times. Cells were digested with trypsin, centrifuged, resuspended, counted and thereafter inoculated into the 24-well plate at a density of $1 \times 10^{4} /$ well. When glioma cells grew to a suitable density, the supernatant was discarded, and the cells were washed 3 times with PBS, fixed with $4 \%$ paraformaldehyde at room temperature for $30 \mathrm{~min}$, washed with PBS 3 times again and stored in a $4^{\circ} \mathrm{C}$ refrigerator. The prepared cover glasses were rinsed with PBS and hydrated 3 times (5 min each time). Each cover glass was treated with approximately $500 \mu \mathrm{l}$ of Triton X-100 (0.3\%) for 20 min and washed with 
PBS 3 times (5 min each time). Afterwards, $100 \mu \mathrm{l}$ of FITC-labelled phalloidin working solution was dropped onto each cover glass for 30 min of staining at room temperature. Then the cells were washed with PBS 3 times ( 5 min each time). After addition of $500 \mu$ of DAPI working solution into each well, the cells were incubated for $10 \mathrm{~min}$ at room temperature, and rinsed with PBS 3 times ( 5 min each time). The cover glasses were thereafter mounted with an appropriate amount of fluorescence quencher, and stored at $4^{\circ} \mathrm{C}$ in a dark wet box. Lamellar pseudopodia and filopodia were observed by fluorescence microscopy or confocal laser scanning microscopy.

\section{Establishment of the mouse model with orthotopically transplanted glioma cells overexpressing CMG2}

CMG2-overexpressed glioma cells and control cells in the logarithmic growth phase were collected, with the density adjusted to $2 \times 10^{4} / \mu$ l. Four- to six-week-old male immunodeficient mice were anesthetized with pentobarbital sodium, and intracranially injected with $5 \mu$ l of cell suspension by using a microsyringe at about $0.5 \mathrm{~cm}$ from the posterior right side of junction between the anterior midline and outer canthus. The needle was inserted by approximately $0.5 \mathrm{~cm}$. The mice were divided into a CMG2 overexpression group and a control group receiving cells transfected with blank vector $(n=5)$. The invasive ability of orthotopically transplanted tumours in vivo was detected, and their effects on the survival time of mice were assessed. After the mice died naturally, their brain tissues were collected timely and prepared into paraffin sections.

\section{HE staining}

The samples of orthotopically transplanted tumours were routinely fixed with formalin, embedded with paraffin, and sectioned into $4 \mu \mathrm{m}$-thick. Paraffin sections were baked at $60^{\circ} \mathrm{C}$ for $30 \mathrm{~min}$ and deparaffinized in xylene 3 times ( 5 min each time). Subsequently, the sections were soaked in 100\%, $95 \%, 85 \%$ and $75 \%$ ethanol solutions successively (5 min each time), rinsed 3 times with PBS (5 min each time), stained with hematoxylin solution for about $1 \mathrm{~min}$, rinsed with deionized water 3 times (5 min each time), and differentiated with hydrochloric acid-ethanol (1\%) until the nucleus became clear under the microscope. Then the sections were washed with running water for 20 min, rinsed with deionized water for about 3 min, stained with eosin solution for $1 \mathrm{~min}$, and dehydrated with gradient concentrations of ethanol solutions. The sections were soaked in $75 \%, 85 \%, 95 \%$ and $100 \%$ ethanol solutions successively ( 5 min each time), and thereafter in xylene twice (10 min each time). Finally, the sections were mounted with an appropriate amount of neutral resin, and observed under the microscope.

\section{Statistical analysis}

All data were analysed by Graphpad Prism 6.0 and SPSS 19.0. The means of multiple groups were compared by one-way analysis of variance, and those of two groups were compared with the independent samples $t$ test. The survival time of tumour-bearing mice was studied by Kaplan-Meier survival analysis.

\section{Results \\ Construction of glioma cell line stably overexpressing CMG2}

The lentivirus carrying the CMG2 gene coding region fragment was infected with glioma cell line U87 cells and U251 primary cells, to obtain cell lines with a stably high expression of CMG2 after combined puromycin drug screening and flow sorting. The results of RT-PCR showed that the mRNA expression of 487 overexpressing cells (U87 OE) was up-regulated by 10 -fold $(p<0.001)$ compared with the CMG2 of the control group, and that of U251 overexpressing cells (U251 OE) increased more significantly, reaching 60 times $(p<0.001)$ (Fig. 1A). The results of Western blot confirmed that the expression levels of CMG2 protein in U87 OE and U251 OE cells were significantly up-regulated $(p<0.001)$ (Fig. 1B).

\section{Overexpression of CMG2 promoted glioma cell invasion and migration}

Invasion and migration experiments were performed on U87 and U251 cells overexpressing CMG2 and their corresponding control cells, respectively. The invasive ability of CMG2-overexpressing U87 cells was significantly higher than that of control cells in vitro ( $p<0.001)$ (Fig. 2A). The scratch assay showed that the migration ability of U87 cells overexpressing CMG2 was significantly enhanced at $12 \mathrm{~h}(p<0.01)$ (Fig. 2B). Similar results were obtained for U251 cells. With the up-regulation of CMG2 expression, both the 
A

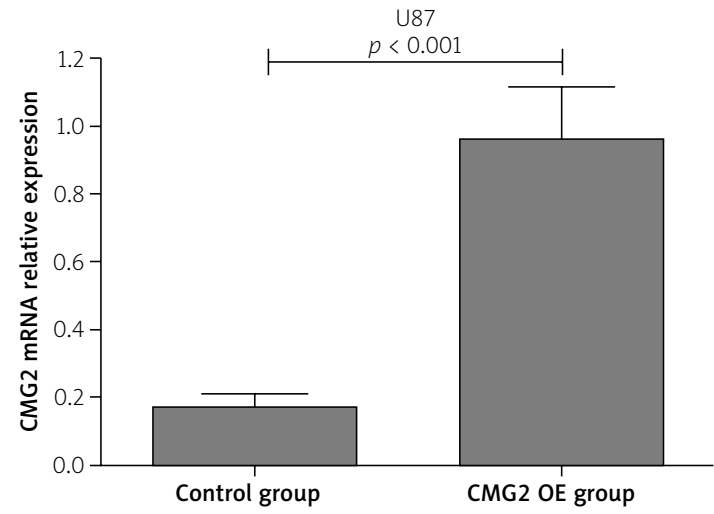

B
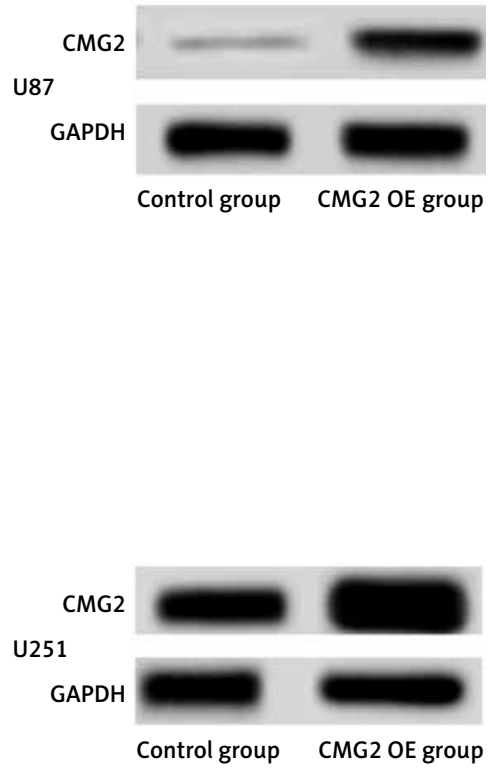
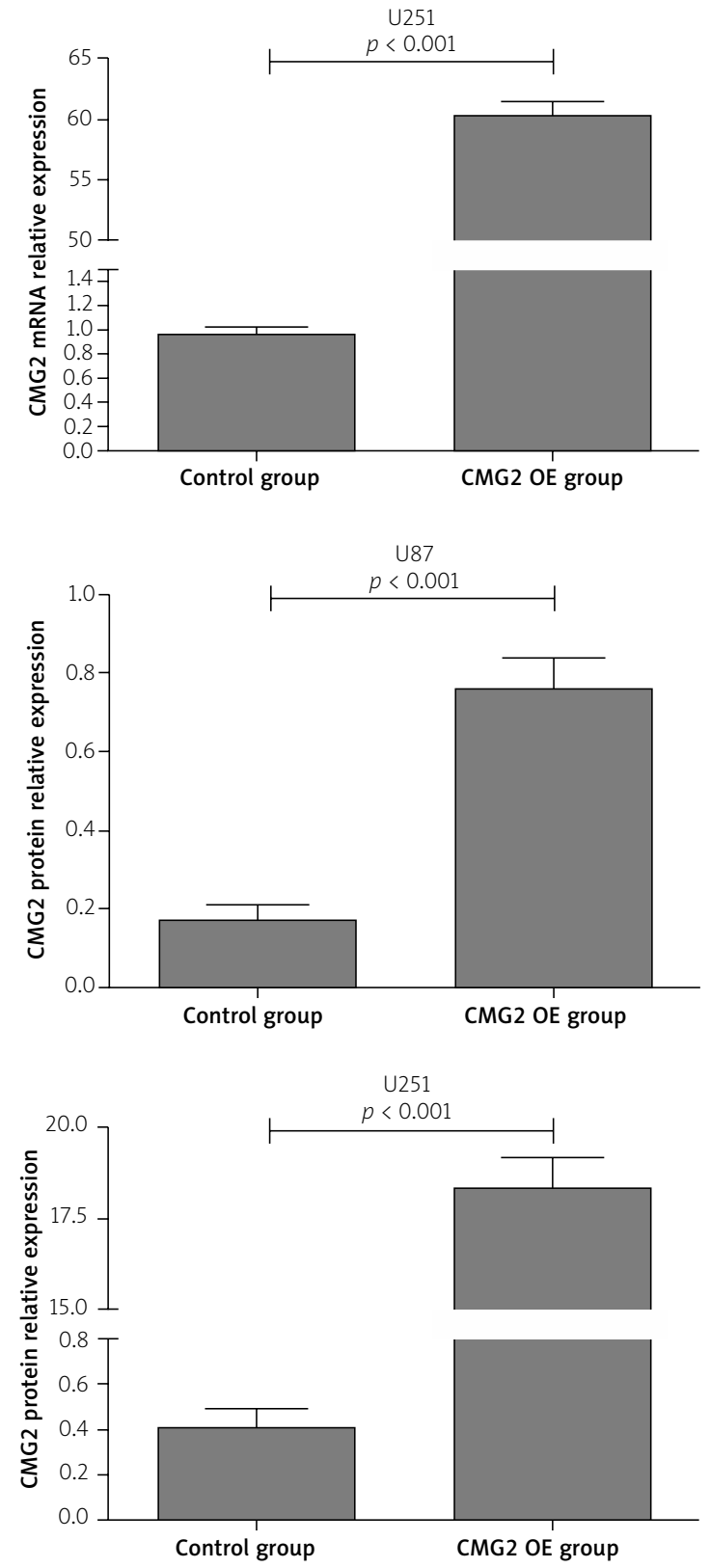

Fig. 1. CMG2 expressions in different groups. A) CMG2 mRNA expressions detected by RT-PCR. Comparison between control and CMG2 overexpression groups, $p<0.001$; B) CMG2 protein expressions detected by Western blot. Comparison between control and CMG2 overexpression groups, $p<0.001$.

invasion $(p<0.001)$ (Fig. 2A) and migration capacities $(p<0.001)$ (Fig. 2B) were enhanced.

\section{Overexpression of CMG2 promoted tumour invasion and shortened survival time in the in vivo model}

We used U251 cells and control cells that expressed CMG2 to perform experiments of orthot- opic transplantation tumour in immunodeficient mice to further observe the effect of CMG2 on the invasive ability of glial cells in vivo. A total of 10 mice were divided into two groups $(n=5)$. The survival time of the mice was recorded until natural death, and the brain tissue sections of the mice were stained with HE. The orthotopic transplantation tumour formed by CMG2-overexpressing cells showed more obvious invasion at the edge of the tumour than the control 

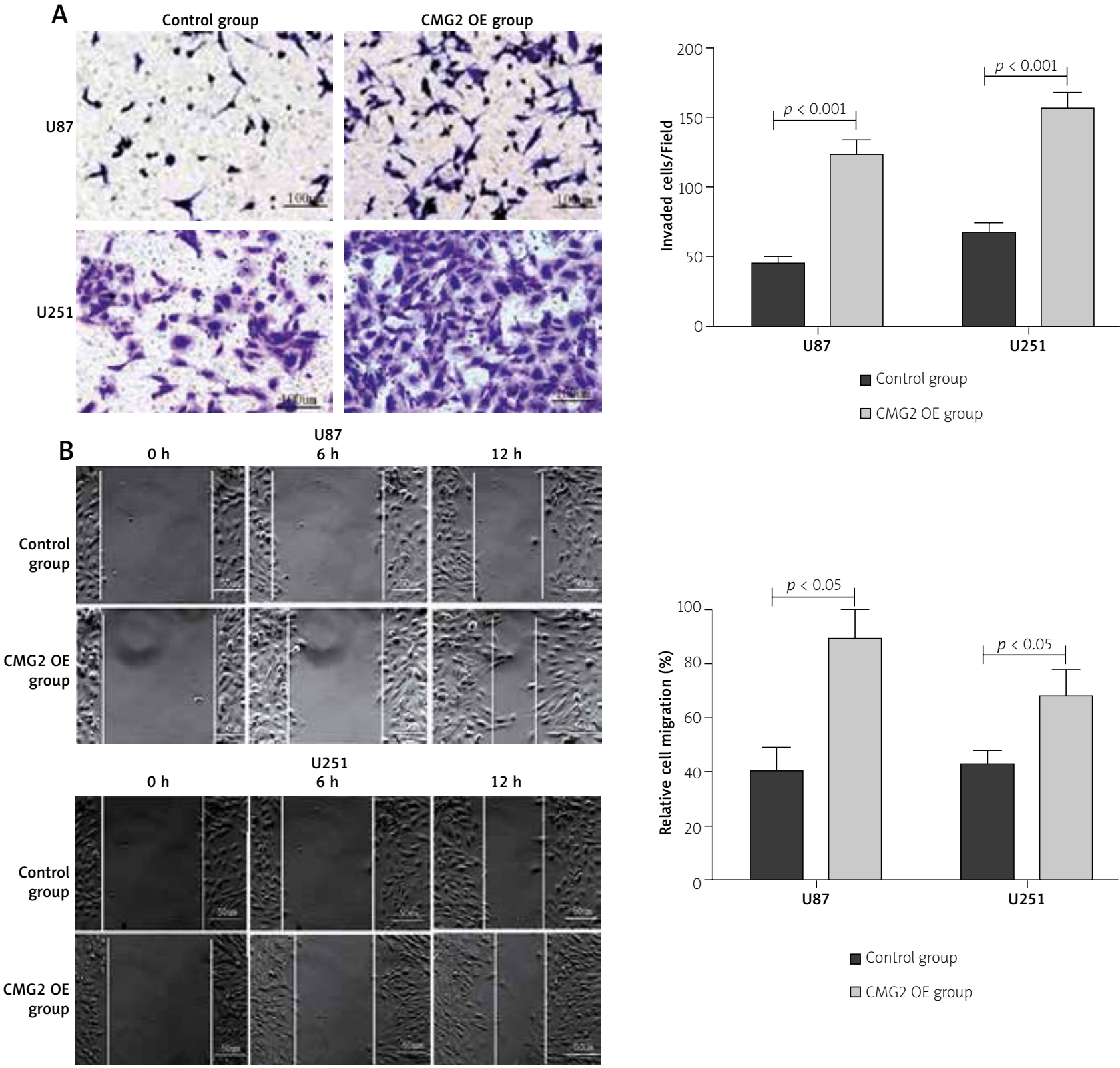

$\square$ CMG2 OE group

Fig. 2. Effects of CMG2 overexpression on glioma cells. A) Overexpression of CMG2 promoted glioma cell invasion detected by the transwell assay. Comparison between control and CMG2 overexpression groups, $p<0.001$; B) overexpression of CMG2 promoted glioma cell migration detected by the scratch assay. Comparison between control and CMG2 overexpression groups, $p<0.05$.

group (Fig. 3A). The survival time of the mice with CMG2-overexpressing cells was significantly shorter than that of control mice ( $p=0.0167$ ) (Fig. 3B).

\section{Overexpression of CMG2 facilitated pseudopodium formation of glioma cells}

We used cellular immunofluorescence staining to analyse the changes in cell morphology of glioma cells
U251 with CMG2 overexpression and control cells (Fig. 4A). The expressed CMG2 carried EGFP green fluorescent protein, i.e. green fluorescence represented the expression of CMG2 (Fig. 4B), and red fluorescence was the cytoskeletal protein F-actin (Fig. 4C). By observation under a laser confocal microscope, we found that CMG2 is present in the cytoplasm in addition to the glioma cell membrane (Fig. 4D). This also suggests that CMG2 overexpression in gliomas may 
A

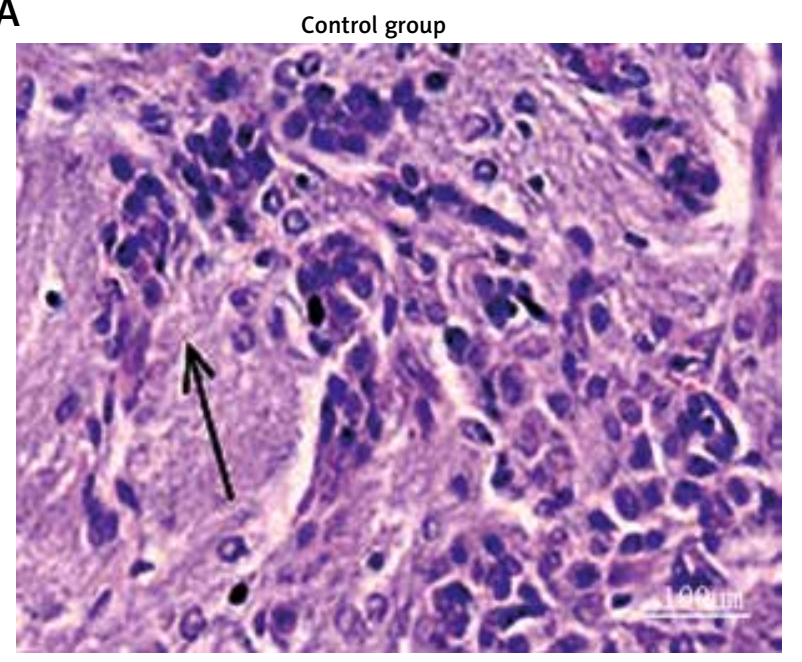

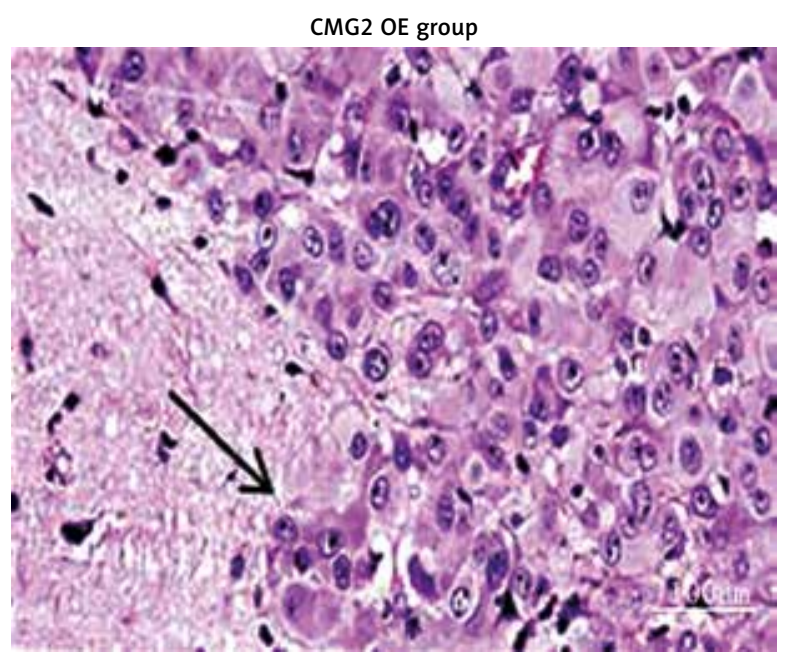

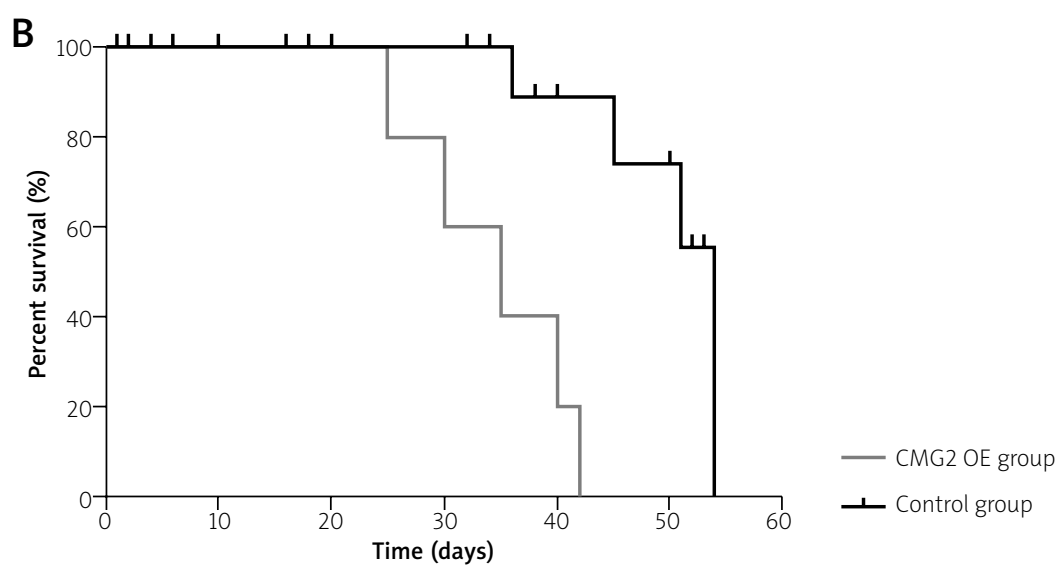

Fig. 3. Effects of CMG2 overexpression on glioma in vivo. A) Overexpression of CMG2 promoted tumour invasion. Arrowheads in representative images indicate invasion frontiers. Magnification: $\times 200$; B) overexpression of CMG2 shortened survival time. Comparison between control and CMG2 overexpression groups, $p<0.05$.

be involved in other biological functions of the cells in addition to its function as a membrane receptor. At the same time, after further observation, we found that cells expressing CMG2 can extend more filopodia and lamellipodia (Fig. 4E).

\section{Overexpression of CMG2 affected YAP expression in the Hippo signalling pathway}

The formation of pseudopodia in tumour cells has been shown to involve complex signalling pathways and molecular mechanisms. The Hippo/YAP pathway is an important pathway regulating the formation of pseudopodia in tumour cells. Accordingly, we attempted to analyse whether CMG2 regulates the formation of pseudopodia in gliomv cells by affecting the Hippo/YAP pathway. YAP is the final effector of the Hippo/YAP pathway. Therefore, we examined the effect of overexpression of CMG2 on YAP expression in U251 cells. The restlts showed that CMG2 overexpression caused a significant up-regulation of total YAP expression, accompanied by a significant down-regulation of phosphorylated YAP that was susceptible to degradation (Fig. 5).

\section{Discussion}

Invasion is the most significant biological feature of malignant glioma. In gliomas, the failure of surgical 

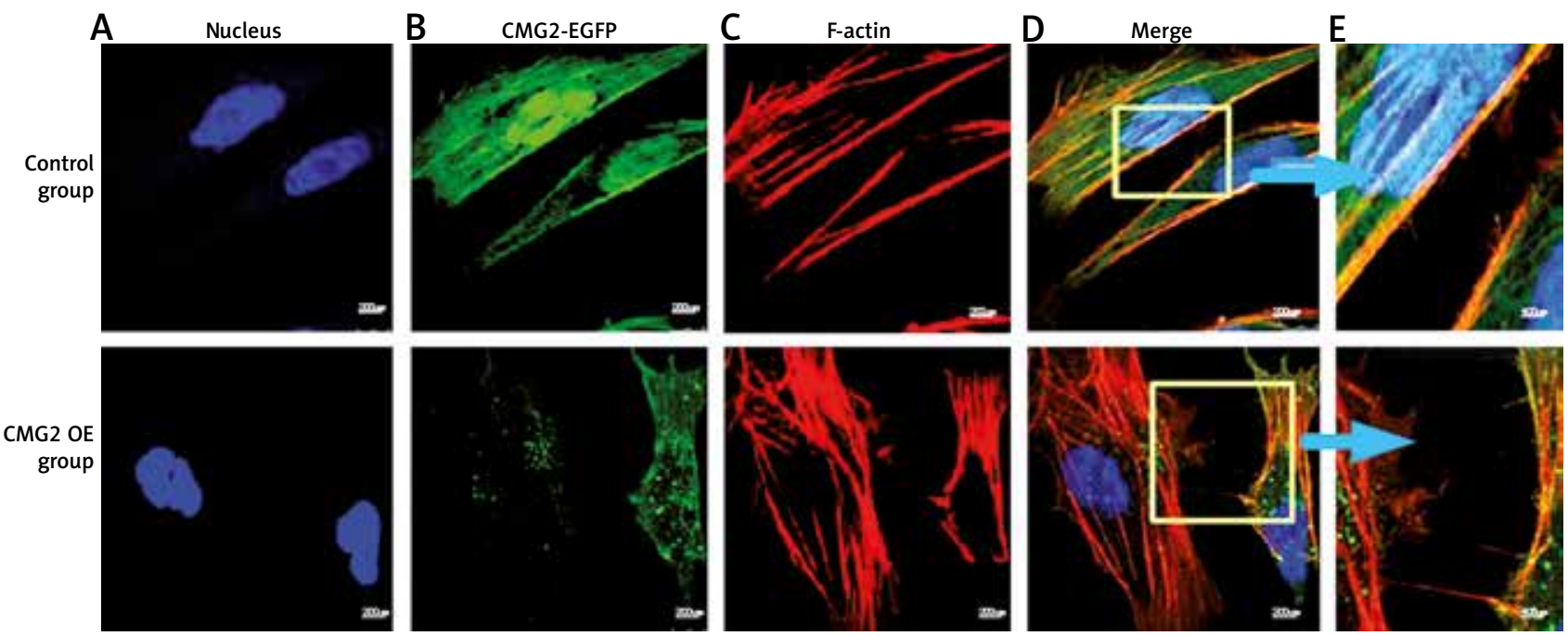

Fig. 4. Immunofluorescence staining results of glioma cells with CMG2 overexpression. A) Cell nucleus; B) CMG2 carrying EGFP green fluorescent protein; C) F-actin protein; D) merged images; E) magnified images of yellow boxes in D. Glioma cells overexpressing CMG2 formed more lamellipodia and filopodia than those of the control group.

A

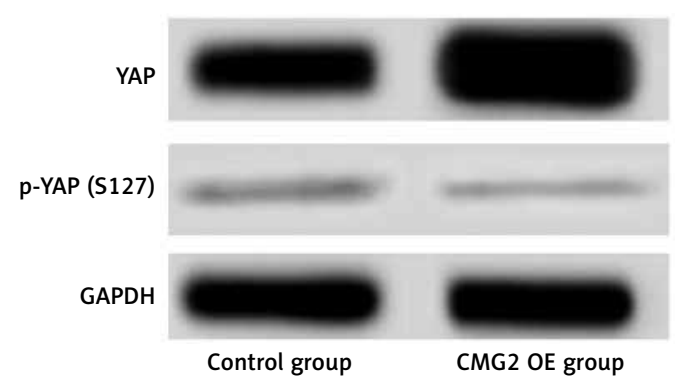

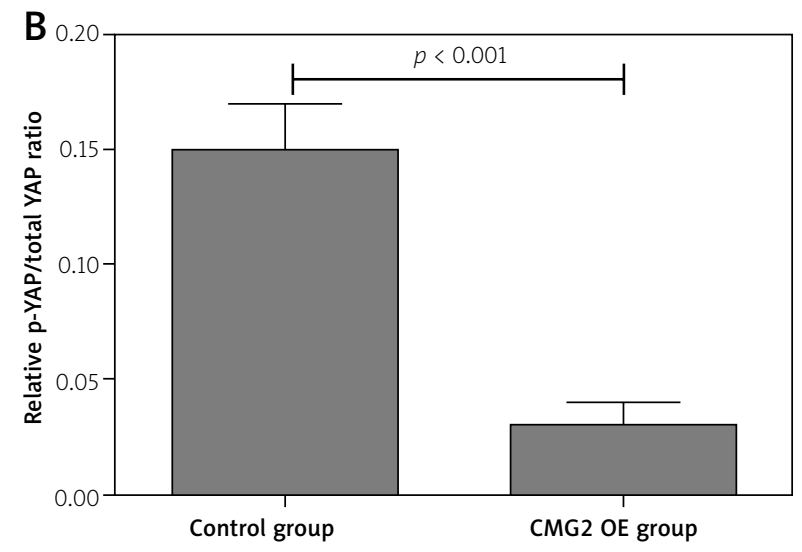

Fig. 5. Effects of CMG2 overexpression on YAP and p-YAP expressions. A) Western blot images; B) relative $p$-YAP/total YAP ratio. Comparison between control and CMG2 overexpression groups, $p<0.001$.

treatment is mainly caused by the high invasiveness of tumour cells. In addition to the special location of the tumour, most anti-tumour drugs cannot reach the tumour site, resulting in poor prognosis of patients with malignant glioma. The median survival time is only about 14 months [18]. Therefore, understanding the mechanism behind the ability to regulate the invasion of glioma cells is of great significance to promoting the diagnosis and treatment of glioma.

It has been more than 10 years since CMG2 was discovered [2]. Although it was discovered in the process of angiogenesis, the previous understanding to $C M G 2$ focused on its role as a receptor-mediated anthrax toxin into host cells to exert toxic effects [21], and mutations in multiple genes can cause a rare but serious hereditary disease, i.e. hyaline fibromatosis syndrome [27]. The study on CMG2 as a receptor-mediated toxin to enter cells focuses on its molecular structure, and mutations in multiple sites have led to the occurrence of the hyaline fibromatosis syndrome, suggesting that it may be associated with abnormal collagen metabolism. In recent years, research on CMG2 has expanded into the field of oncology. Studies have found that CMG2 plays a role in the development of certain tumours. Ye et al. have reported that in prostate cancer, CMG2 did not affect the proliferation of tumour cells, but induced distant metastasis more easily by reducing the adhesion of tumour cells [26]. In breast cancer, CMG2 can inhibit the proliferation of tumour cells and patients with high 
expression of CMG2 have a good prognosis. Although there are few reports of CMG2 in tumours, it can be seen that $C M G 2$ has different biological functions in different tumours. Studies have shown that CMG2 is not expressed in normal brain tissue [21], and glioma is a type of tumour with abnormal angiogenesis. As a vascular development-related molecule, some scholars have found that [5] CMG2 is highly expressed in glioma tissue, and up-regulated with elevated glioma levels, which is associated with poor prognosis. Given that invasion is an important malignant biological behaviour of gliomas and an important factor affecting the prognosis of patients, we are interested in whether CMG2 plays a role in glioma invasion. In this study, we proved that CMG2 was a crucial molecule that promoted the invasion of glioma cells. However, there are few studies on the mechanism of CMG2 biological function in tumours. Next, we will further explore the molecular mechanisms by which CMG2 promotes the invasion of glioma cells.

Based on the evidence that CMG2 promotes the invasion of glioma cells, we further found that the formation of pseudopodia in glioma cells was significantly increased after high expression of CMG2. Pseudopodia are considered to be the most basic organ of mammalian cells [15] and are morphologically expressed as temporary cytoplasmic processes formed during cell migration. When normal cells are transformed into malignant tumour cells, the formation of pseudopodia is increased, and the morphology is also different from that of normal cells, resulting in enhanced cell motility [1]. At present, researchers have endeavoured to detect the changes of tumour cell pseudopodium structure, its role in tumour invasion and metastasis and related regulatory mechanisms. In tumour cells, pseudopodia are classified into lamellipodia, filopodia, podosomes and invadopodia. Among them, the formation of lamellipodia, filopodia, and podosomes involves the reassembly of the actin microfilament skeleton and mainly mediates cell movement; invadopodia mainly degrade the extracellular matrix by secreting matrix metalloproteinase to help the invasion and migration of tumour cells $[15,16]$.

The mechanism for regulating the formation of pseudopodia is numerous and complicated, including Hippo/YAP [3] and PI3K/AKT [11]. However, all of these signalling pathways affect the formation and function of pseudopodia ultimately by affecting the activity of small $G$ protein families such as Rho-GTPase [8]. The Hippo pathway is composed primarily of its core protein kinase cascade reaction chain and its corre- sponding coordinating molecules. The kinase reaction chain is mainly composed of MST1/2 kinase, LATS1/2 kinase, synergistic molecule SAV1 and MOB1 [28]. The protein kinase cascade reaction chain mainly acts on the downstream YAP. As the main effector molecule of this pathway, YAP can be directly phosphorylated by LATS1/2 to p-YAP which is degraded or localized in the cytoplasm. Under normal conditions, activation of this pathway can inhibit tissue growth to maintain the body's proliferation/apoptosis balance and regulate organ size [24]. However, when the Hippo pathway is inhibited, phosphorylation of YAP is reduced and accumulated in the nucleus and combined with transcriptional enhancer associate domain transcription factors to cause expression of a series of genes. Thus, dysregulation of the Hippo pathway and the abnormal expression of YAP can cause cell proliferation/apoptosis imbalance and ultimately lead to tumour formation. Currently, there are many studies on YAP in various tumours [14,22]; in glioma, YAP has been shown to enhance the dryness and invasive ability of glioma cells [9], but the specific mechanism of its effect on glioma cell invasion remains unclear. In triple-negative breast cancer, interference with Rho-GTPase expression can affect the localization of YAP in breast cancer cells, suggesting a mechanism of mutual regulation between YAP and Rho-GTPase in tumours [19]. As a major regulatory family of the cytoskeleton, the regulation between Rho-GTPase and YAP suggests that YAP in tumour cells is highly likely to affect the cytoskeleton and further influence the biological behaviour associated with cytoskeletal proteins. Combined with our results of Western blot, YAP is up-regulated with high expression of $\mathrm{CMG}$, suggesting that CMG2 may regulate cytoskeletal protein by affecting the expression of YAP, which in turn affects the formation of lamellipodia and filopodia in glioma cells.

In summary, CMG2 promotes the invasion of glioma cells, and may induce the formation of pseudopodia by up-regulating YAP expression to promote invasion.

\section{Acknowledgments}

This study was financially supported by the National Natural Science Foundation of China (General Program No. 81571192).

\section{Disclosure}

Authors report no conflict of interest. 


\section{References}

1. Alblazi KM, Siar CH. Cellular protrusions-lamellipodia, filopodia, invadopodia and podosomes-and their roles in progression of orofacial tumours: current understanding. Asian Pac J Cancer Prev 2015; 16: 2187-2191.

2. Bell SE, Mavila A, Salazar R, Bayless KJ, Kanagala S, Maxwell SA, Davis GE. Differential gene expression during capillary morphogenesis in 3D collagen matrices: regulated expression of genes involved in basement membrane matrix assembly, cell cycle progression, cellular differentiation and G-protein signaling. J Cell Sci 2001; 114: 2755-2773.

3. Blom M, Reis K, Heldin J, Kreuger J, Aspenström P. The atypical Rho GTPase RhoD is a regulator of actin cytoskeleton dynamics and directed cell migration. Exp Cell Res 2017; 352: 255-264.

4. Chen KH, Liu S, Leysath CE, Miller-Randolph S, Zhang Y, Fattah R, Bugge TH, Leppla SH. Anthrax toxin protective antigen variants that selectively utilize either the CMG2 or TEM8 receptors for cellular uptake and tumor targeting. I Biol Chem 2016; 291: 22021-22029.

5. Chen R, Cohen AL, Colman H. Targeted therapeutics in patients with high-grade gliomas: past, present, and future. Curr Treat Options Oncol 2016; 17: 42.

6. Dowling O, Difeo A, Ramirez MC, Tukel T, Narla G, Bonafe L, Kayserili H, Yuksel-Apak M, Paller AS, Norton K, Teebi AS, Grum-Tokars V, Martin GS, Davis GE, Glucksman MJ, Martignetti JA. Mutations in capillary morphogenesis gene-2 result in the allelic disorders juvenile hyaline fibromatosis and infantile systemic hyalinosis. Am J Hum Genet 2003; 73: 957-966.

7. El-Kamah GY, Fong K, El-Ruby M, Afifi HH, Clements SE, LaiCheong JE, Amr K, El-Darouti M, McGrath JA. Spectrum of mutations in the ANTXR2 (CMG2) gene in infantile systemic hyalinosis and juvenile hyaline fibromatosis. Br J Dermatol 2010; 163: 213-215.

8. Eves R, Oldham R, Jia L, Mak AS. The roles of akt isoforms in the regulation of podosome formation in fibroblasts and extracellular matrix invasion. Cancers (Basel) 2015; 7: 96-111.

9. Gargini R, Escoll M, García E, García-Escudero R, Wandosell F, Antón IM. WIP drives tumor progression through YAP/TAZ-dependent autonomous cell growth. Cell Rep 2016; 17: 1962-1977.

10. Harvey KF, Zhang X, Thomas DM. The Hippo pathway and human cancer. Nat Rev Cancer 2013; 13: 246-257.

11. Hoshino D, Branch KM, Weaver AM. Signaling inputs to invadopodia and podosomes. J Cell Sci 2013; 126: 2979-2989.

12. Hsu PC, You B, Yang YL, Zhang WQ, Wang YC, Xu Z, Dai Y, Liu S, Yang CT, Li H, Hu B, Jablons DM, You L. YAP promotes erlotinib resistance in human non-small cell lung cancer cells. Oncotarget 2016; 7: 51922-51933.

13. Jiao S, Wang H, Shi Z, Dong A, Zhang W, Song X, He F, Wang Y, Zhang Z, Wang W, Wang X, Guo T, Li P, Zhao Y, Ji H, Zhang L, Zhou Z. A peptide mimicking VGLL4 function acts as a YAP antagonist therapy against gastric cancer. Cancer Cell 2014; 25: 166-180.

14. Juan WC, Hong W. Targeting the Hippo signaling pathway for tissue regeneration and cancer therapy. Genes 2016; 7: pii: E55.

15. Krause M, Gautreau A. Steering cell migration: lamellipodium dynamics and the regulation of directional persistence. Nat Rev Mol Cell Biol 2014; 15: 577-590.
16. Kwiatkowska A, Didier S, Fortin S, Chuang Y, White T, Berens ME, Rushing E, Eschbacher J, Tran NL, Chan A, Symons M. The small GTPase RhoG mediates glioblastoma cell invasion. Mol Cancer 2012; 11: 65

17. Louis DN, Perry A, Reifenberger G, von Deimling A, FigarellaBranger D, Cavenee WK, Ohgaki H, Wiestler OD, Kleihues P, Ellison DW. The 2016 World Health Organization classification of tumors of the central nervous system: a summary. Acta Neuropathol 2016; 131: 803-820.

18. Ostrom QT, Gittleman H, De Blank PM, Finlay JL, Gurney JG, McKean-Cowdin R, Stearns DS, Wolff JE, Liu M, Wolinsky Y, Kruchko C, Barnholtz-Sloan JS. American brain tumor association adolescent and young adult primary brain and central nervous system tumors diagnosed in the United States in 2008-2012. Neuro Oncol 2015; 18: i1-i50.

19. Pascual-Vargas P, Cooper S, Sero J, Bousgouni V, Arias-Garcia M, Bakal C. RNAi screens for Rho GTPase regulators of cell shape and YAP/TAZ localisation in triple negative breast cancer. Sci Data 2017; 4: 170018.

20. Plouffe SW, Hong AW, Guan KL. Disease implications of the Hippo/YAP pathway. Trends Mol Med 2015; 21: 212-222.

21. Scobie HM, Rainey GJ, Bradley KA, Young JA. Human capillary morphogenesis protein 2 functions as an anthrax toxin receptor. Proc Natl Acad Sci U S A 2003; 100: 5170-5174.

22. Janse van Rensburg HJ, Yang $X$. The roles of the Hippo pathway in cancer metastasis. Cell Signal 2016; 28: 1761-1772.

23. Wang Q, Pandol S, Morvaridi S. Sa2058 Hippo-YAP signaling pathway modulates stellate cell activation in pancreatic cancer and pancreatitis. Gastroenterology 2015; 148: S-396.

24. Wang Y, Yu A, Yu FX. The Hippo pathway in tissue homeostasis and regeneration. Protein Cell 2017; 8: 349-359.

25. Yao CB, Zhou X, Chen CS, Lei QY. The regulatory mechanisms and functional roles of the Hippo signaling pathway in breast cancer. Yi Chuan 2017; 39: 617-629.

26. Ye L, Sanders AJ, Sun PH, Mason MD, Jiang WG. Capillary morphogenesis gene 2 regulates adhesion and invasiveness of prostate cancer cells. Oncol Lett 2014; 7: 2149-2153.

27. Youssefian L, Vahidnezhad H, Aghighi Y, Ziaee V, Zeinali S, Abiri M, Uitto J. Hyaline fibromatosis syndrome: a novel mutation and recurrent founder mutation in the CMG2/ANTXR2 gene. Acta Derm Venereol 2017; 97: 108-109.

28. Yu FX, Guan KL. The Hippo pathway: regulators and regulations. Genes Dev 2013; 27: 355-371.

29. Zhao B, Li L, Lei Q, Guan KL. The Hippo-YAP pathway in organ size control and tumorigenesis: an updated version. Genes Dev 2010; 24: 862-874. 